\title{
Thioureas as Ligands in the Pd-Catalyzed Intramolecular Pauson- Khand Reaction
}

Yefeng Tang, ${ }^{\dagger}$ LuJiang Deng, ${ }^{\dagger}$ Yandong zhang, ${ }^{\dagger}$ Guangbin Dong ${ }^{\dagger}, J$ Jahua Chen $^{*}{ }^{\dagger}$ and Zhen Yang $*^{, \dagger}, *$

Key Laboratory of Bioorganic Chemistry and Molecular Engineering of Ministry of Education, College of Chemistry, and Laboratory of Chemical Genomics, ShenZhen Graduate School, Peking University, Beijing, 100871, China, and VivoQuest, Inc. 711 Executive Blvd, Valley Cottage, NY, 10989, USA.

\section{Supporting Information}




\section{General Information}

The boiling point of petroleum ether is between $60-90{ }^{\circ} \mathrm{C}$. Silica gel (200-300 mesh) for purification was purchased from Qing Dao Hai Yang Chemical Industry Co. of China. ${ }^{1} \mathrm{H}-\mathrm{NMR}$ and ${ }^{13} \mathrm{C}$-NMR were recorded at $300 \mathrm{MHz}$ and $75 \mathrm{MHz}$ with Varian Mercury 300 spectrometer or at $400 \mathrm{MHz}$ and $100.6 \mathrm{MHz}$ with Brucker ARX400 spectrometer. Mass spectrometric data were obtained using ZAB-HS mass spectrometer. THF was distilled from sodium, $\mathrm{CH}_{3} \mathrm{CN}$ was distilled from $\mathrm{CaH}_{2}$. Enynes 1, 3a-10a and 12a were prepared according to the references (a) Toshitake Kobayashi, YuJi Koga and Koichi Narasaka, Journal of Organometallic Chemistry, 2001, 624, 73-87, and (b) Krafft, M. E, Romreo, R. H., Scott, I. L., Journal of Organic chemistry, 1992, 57, 5277-5278.

\section{Synthesis of the Substrates for the PKR}

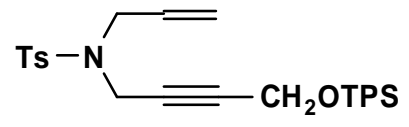

\section{Compound 11a}

\section{N-Allyl-N-(4-(tertbutyldiphenylsilyloxy)but-2-ynyl)4-methylbenzenesulfonamide 11a:}

To a solution of but-2-yn-1,4-diol $(860 \mathrm{mg}, 10 \mathrm{mmol})$ in THF $(50 \mathrm{~mL})$ was added $\mathrm{NaH}$ (240 mg, $10 \mathrm{mmol})$. After stirring for $0.5 \mathrm{~h}$, tert-butylchlorodiphenylsilane $(2.75 \mathrm{~g}, 10 \mathrm{mmol})$ was added to the above solution at $0{ }^{\circ} \mathrm{C}$, and the reaction was then warmed to room temperature gradually and stirred overnight. The reaction was quenched by addition of a saturated solution of $\mathrm{NH}_{4} \mathrm{Cl}(20 \mathrm{~mL})$, and the mixture was extracted with ether $(3 \times 20 \mathrm{~mL})$. The combined organic phase was dried over $\mathrm{Na}_{2} \mathrm{SO}_{4}$. The solvent was removed under vacuum, and the residue was subjected to the next reaction without purification.

To a mixture of N-allyl-4-methyl-benzenesulfonamide (422 mg, $2 \mathrm{mmol}$ ), 4-(tertbutyldiphenylsilyloxy)but-2-yne-1-ol (649 mg, $2 \mathrm{mmol}$ ), and triphenylphosphane (524 mg, 2 mmol) in THF $(15 \mathrm{~mL})$ was added diisopropylazodicarboxylate $(0.42 \mathrm{~mL}, 2 \mathrm{mmol})$ at $0{ }^{\circ} \mathrm{C}$, and the reaction mixture was gradually warmed to room temperature and then stirred overnight. The solvent was removed under vacuum and the residue was purified by flash chromatography on silica gel to give compound 11a $663 \mathrm{mg}$ in $64 \%$ yield. ${ }^{1} \mathrm{H}$ NMR $\left(300 \mathrm{MHz}, \mathrm{CDCl}_{3}\right)$ : $\delta 1.02(\mathrm{~s}, 9 \mathrm{H})$, $2.27(\mathrm{~s}, 3 \mathrm{H}), 3.76(\mathrm{~d}, J=6,6 \mathrm{~Hz}, 2 \mathrm{H}), 4.03(\mathrm{~m}, 4 \mathrm{H}), 5.27(\mathrm{~m}, 2 \mathrm{H}), 5.73(\mathrm{~m}, 1 \mathrm{H}), 7.17(\mathrm{~d}, J=$ $8.4 \mathrm{~Hz}, 2 \mathrm{H}), 7.43$ (m, $6 \mathrm{H}), 7.68$ (m, $2 \mathrm{H}) ;{ }^{13} \mathrm{C} \mathrm{NMR}\left(75 \mathrm{MHz}, \mathrm{CDCl}_{3}\right)$ : $\delta 19.1,21.4,26.6,36.1$, 48.9, 52.3, 77.5, 83.9, 119.8, 127.7, 129.3, 129.8, 131.9, 132.9, 135.4, 135.9, 143.3; LRMS $\left[\mathrm{C}_{30} \mathrm{H}_{35} \mathrm{NO}_{3} \mathrm{SSi}\right]$, (EI) $\mathrm{m} / \mathrm{z}\left(\mathrm{M}^{+}+\mathrm{NH}_{4}\right)$, calcd 535, found 535 .

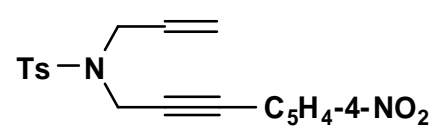

\section{Compound 13a}




\section{N-Allyl-N-(3-(4-nitrophenyl)-propynyl)p -toluenesulfonamide 13a:}

To a mixture of pop-2-yn-1-ol (0.35 mL, $6 \mathrm{mmol})$, 1-iodo-4-nitrobenzene $(1.245 \mathrm{~g}, 5$ $\mathrm{mmol}), \mathrm{PdCl}_{2}\left(\mathrm{PPh}_{3}\right)_{2}(83 \mathrm{mg}, 0.125 \mathrm{mmol})$ and $\mathrm{CuI}(47.5 \mathrm{mg}, 0.25 \mathrm{mmol})$ in dry $\mathrm{CH}_{3} \mathrm{CN}(15 \mathrm{~mL})$ under $\mathrm{N}_{2}$ protection was added $\mathrm{Et}_{3} \mathrm{~N}(2.8 \mathrm{~mL})$ at room temperature, and the reaction was stirred at same temperature for $2 \mathrm{~h}$. The solvent was then removed under vacuum and the was purified by flash chromatograph to give 3-(4-nitrophenyl)-prop-2-yne-1-ol (814 mg) in 92\% yield.

To a mixture of N-allyl-4-methylbenzenesulfonamide $(422 \mathrm{mg}, 2 \mathrm{mmol}$ ), 3-4-nitrophenyl)-prop-2-yne-1-ol (354 mg, $2 \mathrm{mmol}$ ), and triphenyl-phosphane (524 mg, $2 \mathrm{mmol}$ ) in THF $(15 \mathrm{~mL})$ was added diisopropylazodicarboxylate $(0.42 \mathrm{~mL}, 2 \mathrm{mmol})$ a $0{ }^{\circ} \mathrm{C}$, and the reaction mixture was gradually warmed to room temperature, and then stirred overnight. The solvent was removed under vacuum, and the residue was purified on silica gel to give compound 13a (466 mg) in $63 \%$ yield. ${ }^{1} \mathrm{H}$ NMR $\left(300 \mathrm{MHz}, \mathrm{CDCl}_{3}\right): \delta 2.37(\mathrm{~s}, 3 \mathrm{H}), 3.91(\mathrm{~d}, J=6.3 \mathrm{~Hz}, 1 \mathrm{H}), 4.35(\mathrm{~s}, 2 \mathrm{H})$, $5.30(\mathrm{~m}, 2 \mathrm{H}), 5.80(\mathrm{~m}, 1 \mathrm{H}), 7.23(\mathrm{~d}, J=8.7 \mathrm{~Hz}, 2 \mathrm{H}), 7.27(\mathrm{~d}, J=8.4 \mathrm{~Hz}, 2 \mathrm{H}), 7.77(\mathrm{~d}, J=8.4$ $\mathrm{Hz}, 2 \mathrm{H}), 8.14(\mathrm{~d}, J=8.7 \mathrm{~Hz}, 2 \mathrm{H}) ;{ }^{13} \mathrm{C}$ NMR $\left(75 \mathrm{MHz}, \mathrm{CDCl}_{3}\right): \delta 21.3,36.4,49.4,83.6,87.3$, 120.0, 123.2, 127.7, 128.8, 129.5, 131.7, 132.1, 135.7, 143.6, 146.9; LRMS $\left[\mathrm{C}_{19} \mathrm{H}_{18} \mathrm{~N}_{2} \mathrm{O}_{4} \mathrm{~S}\right]$, (EI) $\mathrm{m} / \mathrm{z}\left(\mathrm{M}^{+}\right)$, calcd 370 , found 370 .

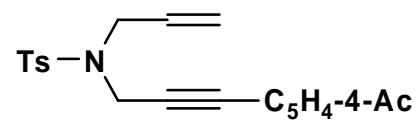

\section{Compound 14a}

\section{$\mathrm{N}$-(2-(4-acetylphenyl)-ethynyl)-N-allyl-4-methylbenzenesulfonamide 14a:}

To a solution of prop-2-yn-1-ol $(0.35 \mathrm{~mL}, 6 \mathrm{mmol}), 4$ '-iodoacetophenone $(1.23 \mathrm{~g}, 5$ $\mathrm{mmol}), \mathrm{PdCl}_{2}\left(\mathrm{PPh}_{3}\right)_{2}(83 \mathrm{mg}, 0.125 \mathrm{mmol})$ and $\mathrm{CuI}(47.5 \mathrm{mg}, 0.25 \mathrm{mmol})$ in dry $\mathrm{CH}_{3} \mathrm{CN}(15 \mathrm{~mL})$ under $\mathrm{N}_{2}$ protection was added $\mathrm{Et}_{3} \mathrm{~N}(2.8 \mathrm{~mL})$ at room temperature, and the reaction mixture was stirred at same temperature for $2 \mathrm{~h}$ The solvent was removed under vacuum, and the residue was purified by flash chromatograph to give 3-(4acetylphenyl)-prop-2-yn-1-ol (744mg) in 93\% yield.

To a mixture of N-allyl4-methyl-benzenesulfonamide (422 mg, $2 \mathrm{mmol}), 3$ - (4-acetylphenyl)-prop-2-yn-1-ol (348 mg, $2 \mathrm{mmol}$ ), and triphenyl-phosphane (524 mg, $2 \mathrm{mmol}$ ) in THF (15 $\mathrm{mL})$ was added diisopropylazodicarboxylate $(0.42 \mathrm{~mL}, 2 \mathrm{mmol})$ at $0{ }^{\circ} \mathrm{C}$, and the reaction mixture was gradually warmed to room temperature, and stirred overnight. The solvent was removed under vacuum and the residue was purified on silica gel to give compound $\mathbf{1 4 a}(418 \mathrm{mg})$ in $57 \%$ yield. ${ }^{1} \mathrm{H}$ NMR $\left(200 \mathrm{MHz}, \mathrm{CDCl}_{3}\right): \delta 2.27(\mathrm{~s}, 3 \mathrm{H}), 2.68(\mathrm{~s}, 1 \mathrm{H}), 3.74(\mathrm{~d}, J=6.6 \mathrm{~Hz}, 2 \mathrm{H}), 4.33$ (s, $2 \mathrm{H}), 5.30(\mathrm{~m}, 2 \mathrm{H}), 5.77(\mathrm{~m}, 1 \mathrm{H}), 7.12(\mathrm{~d}, J=8.6 \mathrm{~Hz}, 2 \mathrm{H}), 7.27(\mathrm{~d}, J=8.2 \mathrm{~Hz}, 2 \mathrm{H}), 7.72(\mathrm{~d}, J=$ $8.2 \mathrm{~Hz}, 2 \mathrm{H}), 7.86(\mathrm{~d}, J=8.4 \mathrm{~Hz}, 2 \mathrm{H}) ;{ }^{13} \mathrm{C}$ NMR $\left(75 \mathrm{MHz}, \mathrm{CDCl}_{3}\right): \delta 21.3,26.5,36.6,49.3,84.8$, $85.2,120.1,127.0,127.7,127.9,128.0,129.6,131.6,132.0,135.9,143.6,197.3$; LRMS $\left[\mathrm{C}_{21} \mathrm{H}_{21} \mathrm{NO}_{3} \mathrm{~S}\right]$, (EI) $\mathrm{m} / \mathrm{z}\left(\mathrm{M}^{+}\right)$, calcd 367, found 367 .

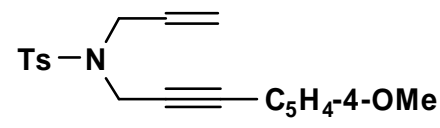

\section{Compound 15a}




\section{N-allyl-N -(2-(4-methoxyphenyl)ethynyl)-4-methylbenzenesulfonamide 15a:}

To a solution of prop-2-yn-1-ol (0.35 mL, $60 \mathrm{mmol}), 1$-methoxy-4-methylbenzene (1.17 g, $5 \mathrm{mmol}), \mathrm{PdCl}_{2}\left(\mathrm{PPh}_{3}\right)_{2}(83 \mathrm{mg}, 0.125 \mathrm{mmol})$, and $\mathrm{CuI}(47.5 \mathrm{mg}, 0.25 \mathrm{mmol})$ in dry $\mathrm{CH}_{3} \mathrm{CN}(15$ $\mathrm{mL})$ was added $\mathrm{Et}_{3} \mathrm{~N}(2.8 \mathrm{~mL})$ at room temperature under $\mathrm{N}_{2}$, and the reaction mixture was stirred for $2 \mathrm{~h}$ The solvent was removed under vacuum, and the residue was purified by flash chromatograph on silica gel to give 2-(4-methoxyphenyl)-ethynol $(729 \mathrm{mg})$ in $90 \%$ yield.

To a solution of N-allyl-4-methyl-benzenesulfonamide(422 mg, $2.0 \mathrm{mmol}), 2-(4-$ methoxyphenyl)-ethynol (324 mg, $2.0 \mathrm{mmol})$, and triphenyl-phosphane $(524 \mathrm{mg}, 2.0 \mathrm{mmol})$ in THF $(15 \mathrm{~mL})$ was added diisopropylazodicarboxylate $(0.42 \mathrm{~mL}, 20 \mathrm{mmol})$ at $0{ }^{\circ} \mathrm{C}$, and the reaction mixture was gradually warmed to room temperature, and then stirred overnight. The solvent was removed under vacuum and the residue was purified by flash chromatography on silica gel to give compound $15 \mathrm{a}(454 \mathrm{mg})$ in $64 \%$ yield. ${ }^{1} \mathrm{H}$ NMR $\left(200 \mathrm{MHz}, \mathrm{CDCl}_{3}\right): \delta 2.36(\mathrm{~s}, 3$ H), $3.79(\mathrm{~s}, 1 \mathrm{H}), 3.90(\mathrm{~d}, J=6.4 \mathrm{~Hz}, 2 \mathrm{H}), 4.29(\mathrm{~s}, 2 \mathrm{H}), 5.23-5.37$ (m, $2 \mathrm{H}), 5.80(\mathrm{~m}, 1 \mathrm{H}), 6.78$ (d, $J=8.6 \mathrm{~Hz}, 2 \mathrm{H}), 6.99$ (d, $J=8.6 \mathrm{~Hz}, 2 \mathrm{H}), 7.24(\mathrm{~d}, J=8.2 \mathrm{~Hz}, 2 \mathrm{H}), 7.76(\mathrm{~d}, J=8.2 \mathrm{~Hz}, 2 \mathrm{H})$; ${ }^{13} \mathrm{C} \mathrm{NMR}\left(50 \mathrm{MHz}, \mathrm{CDCl}_{3}\right): \delta 21.3,36.7,49.1,55.2,80.1,85.5,113.7,114.2,119.9,127.8,129.5$, 132.1, 132.9, 135.9, 143.4, 159.6; LRMS [ $\left.\mathrm{C}_{20} \mathrm{H}_{21} \mathrm{NO}_{3} \mathrm{~S}\right]$, (EI) m/z $\left(\mathrm{M}^{+}\right)$, calcd 355, found 355.

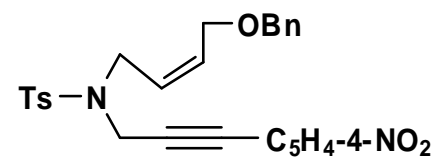

\section{Compound 16a}

\section{(Z)-N-(4-(benzyloxy)but-2-enyl)-4-methyl-N -(3-(4 -nitrophenyl)prop-2-ynyl)-benzenesulfon- amide 16a:}

To a solution of (Z)-but2-ene-1,4-diol $(0.82 \mathrm{~mL}, 10 \mathrm{mmol})$ in THF $(50 \mathrm{~mL})$ was added $\mathrm{NaH}(240 \mathrm{mg}, 10 \mathrm{mmol})$. After stirring for $0.5 \mathrm{~h}$, benzyl bromide $(1.71 \mathrm{~g}, 10 \mathrm{mmol})$ was added at 0 ${ }^{\circ} \mathrm{C}$, and the reaction mixture was warmed to room temperature gradually, and then stirred overnight. The reaction was quenched with a saturated solution of $\mathrm{NH}_{4} \mathrm{Cl}(20 \mathrm{~mL})$, and the mixture was extracted with ether $(3 \times 20 \mathrm{~mL})$. The combined organic phase was dried over $\mathrm{Na}_{2} \mathrm{SO}_{4}$. The solvent was removed under vacuum and the residue was submitted to the next reaction.

To a mixture of N-propargyl4-methyl-benzenesulfonamide (418 mg, 2 mmol), (Z)-3(benzyloxy)prop-1-en-1-ol (356 mg, $2 \mathrm{mmol}$ ) and triphenylphosphane (524 mg, $2 \mathrm{mmol}$ ) in THF $(15 \mathrm{~mL})$ was added diisopropylazodicarboxylate $(0.42 \mathrm{~mL}, 20 \mathrm{mmol})$ at $0{ }^{\circ} \mathrm{C}$, and the reaction mixture was gradually warmed to room temperature, and then stirred overnight. The solvent was removed under vacuum and the residue was purified on silica gel to give terminal acetylene (406 $\mathrm{mg}$ ) in $55 \%$ yield.

The above generated terminal acetylene $(739 \mathrm{mg}, 20 \mathrm{mmol}), 1$-iodo-4-nitrobenzene (498 $\mathrm{mg}, 2 \mathrm{mmol}), \mathrm{PdCl}_{2}\left(\mathrm{PPh}_{3}\right)_{2}(33 \mathrm{mg}, 0.05 \mathrm{mmol})$ and $\mathrm{CuI}(19 \mathrm{mg}, 0.1 \mathrm{mmol})$ were dissolved in dry $\mathrm{CH}_{3} \mathrm{CN}(15 \mathrm{~mL})$. To this solution was added $\mathrm{Et}_{3} \mathrm{~N}(1.2 \mathrm{~mL})$ at room temperature under $\mathrm{N}_{2}$ and the reaction mixture was stirred at the same temperature for $2 \mathrm{~h}$ The solvent was removed under vacuum and the residue was purified by flash chromatograph on silica gel to give product 16a $(911 \mathrm{mg})$ in $93 \%$ yield. ${ }^{1} \mathrm{H}$ NMR $\left(300 \mathrm{MHz}, \mathrm{CDCl}_{3}\right): \delta 2.35(\mathrm{~s}, 3 \mathrm{H}), 3.95(\mathrm{~d}, J=7.2 \mathrm{~Hz}, 1 \mathrm{H}), 4.11$ $(\mathrm{d}, J=7.2 \mathrm{~Hz}, 2 \mathrm{H}), 4.33(\mathrm{~s}, 2 \mathrm{H}), 4.43(\mathrm{~s}, 2 \mathrm{H}), 5.61(\mathrm{~m}, 1 \mathrm{H}), 5.91(\mathrm{~m}, 1 \mathrm{H}), 7.14(\mathrm{~d}, J=8.1 \mathrm{~Hz}$, 
$2 \mathrm{H}), 7.27(\mathrm{~m}, 7 \mathrm{H}), 7.77(\mathrm{~d}, J=8.1 \mathrm{~Hz}, 2 \mathrm{H}), 8.10(\mathrm{~d}, J=8.4 \mathrm{~Hz}, 2 \mathrm{H}) ;{ }^{13} \mathrm{C}$ NMR $(75 \mathrm{MHz}$, $\left.\mathrm{CDCl}_{3}\right): \delta 21.4,36.6,43.6,65.4,72.4,83.7,87.5,123.3,126.2,127.4,127.6,127.8,128.3,128.7$, 129.5, 132.0, 132.1, 135.6, 137.7, 143.6, 147.0; LRMS $\left[\mathrm{C}_{27} \mathrm{H}_{26} \mathrm{~N}_{2} \mathrm{O}_{5} \mathrm{~S}\right],(\mathrm{EI}) \mathrm{m} / \mathrm{z}\left(\mathrm{M}^{+}\right)$, calcd 490, found 490 .

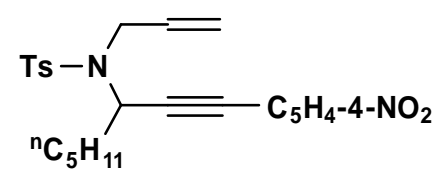

\section{Compound 17a}

\section{$\mathrm{N}$-allyl4 -methyl -N-(1 -(4-nitrophenyl)-oct-1-yn-3 -yl)-benzenesulfonamide 17a:}

To a mixture of $\mathrm{N}$-ally-4-methyl-benzenesulfonamide (1.055 mg, $5 \mathrm{mmol})$, oct-1-yn-3-ol $(0.73 \mathrm{~mL}, 5 \mathrm{mmol})$, and triphenyphosphane $(1.31 \mathrm{mg}, 5 \mathrm{mmol})$ in THF $(50 \mathrm{~mL})$ was added diisopropylazodicarboxylate $(1.05 \mathrm{~mL}, 5 \mathrm{mmol})$ a $0{ }^{\circ} \mathrm{C}$, and the reaction mixture was gradually warmed to room temperature, and then stirred overnight. The solvent was removed under vacuum and the residue was purified by flash chromatography on silica gel to give the alcohol $(831 \mathrm{mg})$ in $52 \%$ yield.

The generated alcohol above (639 mg, $2 \mathrm{mmol}$ ), 1-iodo-4-nitrobenzene (498 mg, $2 \mathrm{mmol}$ ), $\mathrm{PdCl}_{2}\left(\mathrm{PPh}_{3}\right)_{2}(33 \mathrm{mg}, 0.05 \mathrm{mmol})$, and $\mathrm{CuI}(19 \mathrm{mg}, 0.1 \mathrm{mmol})$ were added to dry $\mathrm{CH}_{3} \mathrm{CN}(15 \mathrm{~mL})$ at room temperature under $\mathrm{N}_{2}$. To this solution was added $\mathrm{Et}_{3} \mathrm{~N}(1.2 \mathrm{~mL})$, and the reaction mixture was stirred for $2 \mathrm{~h}$ at the same temperature. The solvent was removed under vacuum and the residue was purified by flash chromatograph on silica gel to give compound $\mathbf{1 7 a}(819 \mathrm{mg})$ in $93 \%$ yield. ${ }^{1} \mathrm{H}$ NMR $\left(200 \mathrm{MHz}, \mathrm{CDCl}_{3}\right): \delta 0.90(\mathrm{~m}, 3 \mathrm{H}), 1.21-1.56(\mathrm{~m}, 6 \mathrm{H}), 1.80(\mathrm{~m}, 2 \mathrm{H}), 2.37(\mathrm{~s}, 3$ $\mathrm{H}), 3.75(\mathrm{dd}, J=6.8 \mathrm{~Hz}, 1 \mathrm{H}), 3.98(\mathrm{~m}, 1 \mathrm{H}), 4.91(\mathrm{t}, J=7.8 \mathrm{~Hz}, 1 \mathrm{H}), 5.12-5.31(\mathrm{~m}, 2 \mathrm{H}), 5.98(\mathrm{~m}$, $1 \mathrm{H}), 7.19(\mathrm{~d}, J=8.6 \mathrm{~Hz}, 2 \mathrm{H}), 7.25(\mathrm{~d}, J=8.2 \mathrm{~Hz}, 2 \mathrm{H}), 7.80(\mathrm{~d}, J=8.2 \mathrm{~Hz}, 2 \mathrm{H}), 8.13(\mathrm{~d}, J=8.6$ $\mathrm{Hz}, 2 \mathrm{H}) ;{ }^{13} \mathrm{C} \mathrm{NMR}\left(50 \mathrm{MHz}, \mathrm{CDCl}_{3}\right): \delta 13.9,21.4,22.4,25.6,30.9,34.9,47.8,51.5,83,5,91.7$, 117.1, 123.3, 127.8, 129.0, 129.4, 132.1, 136.0, 143.4, 146.9; LRMS $\left[\mathrm{C}_{24} \mathrm{H}_{28} \mathrm{~N}_{2} \mathrm{O}_{4} \mathrm{~S}\right],(\mathrm{EI}) \mathrm{m} / \mathrm{z}$ $\left(\mathrm{M}^{+}\right)$, calcd 440, found 440 .

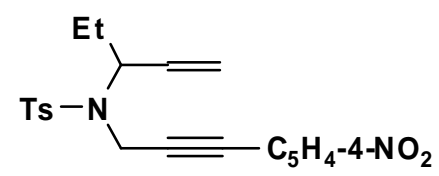

\section{Compound 18a}

\section{4-methyl -N-(3 -(4-nitrophenyl)prop-2-ynyl)-N-(pent-1-en-3-yl)-benzenesulfonamide 18a:}

To a mixture of N-propargyl-4-methyl-benzenesulfonamide (1.045 g, $5 \mathrm{mmol}$ ), pent-1-en3-ol (0.51 mL, $5 \mathrm{mmol})$, and triphenyl-phosphane $(1.31 \mathrm{~g}, 5 \mathrm{mmol})$ was added to THF $(50 \mathrm{~mL})$. To this solution was added diisopropylazodicarboxylate $(1.05 \mathrm{~mL}, 5 \mathrm{mmol})$ at $0{ }^{\circ} \mathrm{C}$, and the reaction mixture was gradually warmed to room temperature, and the stirred overnight. The solvent was removed under vacuum and the residue was purified by flash chromatography on silica gel to give the primary alcohol $(0.8 \mathrm{~g})$ in $58 \%$ yield.

The primary alcohol made above (554 mg, $2 \mathrm{mmol}$ ), 1-iodo-4-nitrobenzene $(498 \mathrm{mg}, 2$ 
mmol), $\mathrm{PdCb}\left(\mathrm{PPh}_{3}\right)_{2}(33 \mathrm{mg}, 0.05 \mathrm{mmol})$, and $\mathrm{CuI}(19 \mathrm{mg}, 0.1 \mathrm{mmol})$ were added to dry $\mathrm{CH}_{3} \mathrm{CN}$ $(15 \mathrm{~mL})$ under $\mathrm{N}_{2}$. To this solution was added $\mathrm{Et}_{3} \mathrm{~N}(1.2 \mathrm{~mL})$ at room temperature, and reac tion mixture was stirred at same temperature for $2 \mathrm{~h}$. The solvent was removed under vacuum and the residue was purified by flash chromatograph on silica gel to give $18 \mathbf{a}(717 \mathrm{mg})$ in $90 \%$ yield. ${ }^{1} \mathrm{H}$ NMR (200MHz, $\left.\mathrm{CDCl}_{3}\right): \delta 0.93(\mathrm{t}, J=7.2 \mathrm{~Hz}, 3 \mathrm{H}), 1.72(\mathrm{~m}, 2 \mathrm{H}), 2.35(\mathrm{~s}, 3 \mathrm{H}), 4.28(\mathrm{~d}+\mathrm{d}, J=$ $13.0 \mathrm{~Hz}, 2 \mathrm{H}), 4.37$ (m, $1 \mathrm{H}), 5.19(\mathrm{~m}, 2 \mathrm{H}), 5.73(\mathrm{~m}, 1 \mathrm{H}), 7.26(\mathrm{~d}, J=8.6 \mathrm{~Hz}, 2 \mathrm{H}), 7.36$ (d, $J=$ $8.2 \mathrm{~Hz}, 2 \mathrm{H}), 7.78(\mathrm{~d}, J=8.2 \mathrm{~Hz}, 2 \mathrm{H}), 8.16(\mathrm{~d}, J=8.6 \mathrm{~Hz}, 2 \mathrm{H}) ;{ }^{13} \mathrm{C} \mathrm{NMR}\left(50 \mathrm{MHz}, \mathrm{CDCl}_{3}\right): \delta$ $10.9,21.2,24.5,32.8,61.3,81.9,90.9,117.7,123.1,127.3,127.6$; LRMS $\left[\mathrm{C}_{21} \mathrm{H}_{22} \mathrm{~N}_{2} \mathrm{O}_{4} \mathrm{~S}\right]$, (EI) $\mathrm{m} / \mathrm{z}\left(\mathrm{M}^{+}\right)$, calcd 398, found 398 .

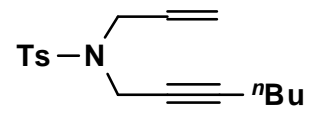

\section{Compound 19a}

To a mixture of N-ally-4-methyl-benzenesulfonamide (553 mg, $5 \mathrm{mmol}$ ), hept-2-yn-1-ol (560 mg, $5 \mathrm{mmol})$, and triphenylphosphane $(1.31 \mathrm{mg}, 5 \mathrm{mmol})$ in THF $(50 \mathrm{~mL})$ was added diisopropylazodicarboxylate $(1.05 \mathrm{~mL}, 5 \mathrm{mmol})$ at $0{ }^{\circ} \mathrm{C}$, and the reaction mixture was gradually warmed to room temperature, and then stirred overnight. The solvent was removed under vacuum and the residue was purified by flash chromatography on silica gel to give 19a (794 mg) in 52\% yield. ${ }^{1} \mathrm{H}$ NMR (200MHz, $\left.\mathrm{CDCl}_{3}\right): \delta 0.87(\mathrm{~m}, 3 \mathrm{H}), 1.25(\mathrm{~m}, 4 \mathrm{H}), 1.90(\mathrm{~m}, 2 \mathrm{H}), 2.42(\mathrm{~s}, 3 \mathrm{H})$, $3.79(\mathrm{~d}, J=6.2 \mathrm{~Hz}, 2 \mathrm{H}), 4.14(\mathrm{~s}, 2 \mathrm{H}), 5.31(\mathrm{~m}, 2 \mathrm{H}), 5.82(\mathrm{~m}, 1 \mathrm{H}) ;{ }^{13} \mathrm{C} \mathrm{NMR}\left(50 \mathrm{MHz}, \mathrm{CDCl}_{3}\right)$ : $\delta$ 13.4, 17.9, 21.3, 21.6, 30.3, 36.2, 487, 74.2, 86.2, 119.6, 127.8, 129.3, 132.2, 136.2, 143.2; LRMS $\left[\mathrm{C}_{17} \mathrm{H}_{23} \mathrm{NO}_{2} \mathrm{~S}\right],(\mathrm{EI}) \mathrm{m} / \mathrm{z}\left(\mathrm{M}^{+}\right)$, calcd 305, found 305.

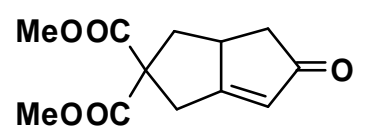

\section{Compound 2}

To a solution of $\mathrm{PdCl}_{2}(13.3 \mathrm{mg}, 0.075 \mathrm{mmol})$, tmtu $(9.9 \mathrm{mg}, 0.075 \mathrm{mmol})$ in dry THF (1 $\mathrm{mL})$ under a balloon pressure of $\mathrm{CO}$ was added substrate $\mathbf{1}(105 \mathrm{mg}, 0.5 \mathrm{mmol})$ in THF $(10 \mathrm{~mL})$, and reaction mixture was stirred under a balloon pressure of $\mathrm{CO}$ at $50{ }^{\circ} \mathrm{C}$ for $24 \mathrm{~h}$. The solvent was removed under vacuum and the residue was purified by flash chromatograph on silica gel (first elution with petroleum ether/ethyl acetate $=6 / 1$, then with petrol ether/ethyl acetate $=2 / 1$ ) to give compound $2(86 \mathrm{mg})$ in $72 \%$ yield. ${ }^{1} \mathrm{H} N \mathrm{NM}\left(300 \mathrm{MHz}, \mathrm{CDCl}_{3}\right): \delta 1.73(\mathrm{t}, J=12.6 \mathrm{~Hz}, 1 \mathrm{H})$, $2.10(\mathrm{dd}, J=3.3,18 \mathrm{~Hz}, 1 \mathrm{H}), 2.65(\mathrm{dd}, J=6.6, J=18 \mathrm{~Hz}, 1 \mathrm{H}), 2.82(\mathrm{dd}, J=7.8, J=12.9 \mathrm{~Hz}, 1$ H), $3.12(\mathrm{~m}, 1 \mathrm{H}), 3.26(\mathrm{~d}, J=18.9 \mathrm{~Hz}, 1 \mathrm{H}), 3.36(\mathrm{~d}, J=18.9 \mathrm{~Hz}, 1 \mathrm{H}), 3.73(\mathrm{~s}, 3 \mathrm{H}), 3.79$ (s, 3 $\mathrm{H}), 5.92(\mathrm{dd}, J=1.5,3.6 \mathrm{~Hz}, 1 \mathrm{H}) ;{ }^{13} \mathrm{C} \mathrm{NMR}\left(75 \mathrm{MHz}, \mathrm{CDCl}_{3}\right): \delta 35.1,38.9,41.9,44.9,53.1,53.2$, 60.6, 125.5, 171.1, 171.8, 185.2, 209.3; LRMS $\left[\mathrm{C}_{12} \mathrm{H}_{14} \mathrm{O}_{5}\right]$, (EI) $\mathrm{m} / \mathrm{z}\left(\mathrm{M}^{+}\right)$, calcd 238, found 238.

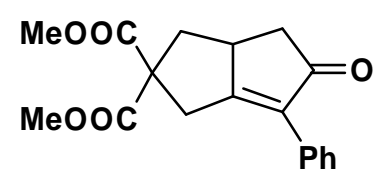

\section{Compound 4b}


To a solution of $\mathrm{PdCl}_{2}(13.3 \mathrm{mg}, 0.075 \mathrm{mmol})$, tmtu (9.9 mg, $\left.0.075 \mathrm{mmol}\right)$ in dry THF (1 $\mathrm{mL})$ under a balloon pressure of $\mathrm{CO}$ was added substrate $\mathbf{4 a}(143 \mathrm{mg}, 0.5 \mathrm{mmol})$ in THF (10mL), and the reaction mixture was stirred at $50{ }^{\circ} \mathrm{C}$ for $24 \mathrm{~h}$. The solvent was removed under vacuum and the residue was purified by flash chromatograph on silica gel (first elution with petroleum ether/ethyl acetate $=6 / 1$, then with petrol ether/ethyl acetate $=2 / 1)$ to give product $\mathbf{4 b}(38 \mathrm{mg})$ in $24 \%$ yield. ${ }^{1} \mathrm{H}$ NMR $\left(300 \mathrm{MHz}, \mathrm{CDCl}_{3}\right): \delta 1.76(\mathrm{t}, J=12.9 \mathrm{~Hz}, 1 \mathrm{H}), 2.30(\mathrm{dd}, J=3.3,18.0 \mathrm{~Hz}, 1$ H), $2.80(\mathrm{dd}, J=12.9 \mathrm{~Hz}, 1 \mathrm{H}), 2.86(\mathrm{t}, J=12.9 \mathrm{~Hz}, 1 \mathrm{H}), 3.14(\mathrm{~m}, 1 \mathrm{H}), 3.30(\mathrm{~d}, J=18.5 \mathrm{~Hz}, 1 \mathrm{H})$, $3.64(\mathrm{~d}, J=18.5 \mathrm{~Hz}, 1 \mathrm{H}), 3.74(\mathrm{~s}, 3 \mathrm{H}), 3.90(\mathrm{~s}, 3 \mathrm{H}), 7.58-7.28(\mathrm{~m}, 5 \mathrm{H}) ;{ }^{13} \mathrm{C} \mathrm{NMR}(75 \mathrm{MHz}$, $\left.\mathrm{CDCl}_{3}\right): \delta 35.8,38.7,42.4,42.4,42.7,52.9,53.1,61.0,128.1,128.3,128.4,130.8,135.4,171.1$, 172.0, 178.7, 207.0; LRMS [ $\left.\mathrm{C}_{18} \mathrm{H}_{18} \mathrm{O}_{5}\right]$, (EI) m/z $\left(\mathrm{M}^{+}\right)$, calcd 314, found 314 .

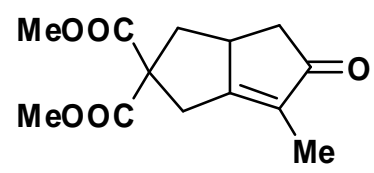

\section{Compound 5b}

To a solution of $\mathrm{PdCl}_{2}(13.3 \mathrm{mg}, 0.075 \mathrm{mmol})$, tmtu $(9.9 \mathrm{mg}, 0.075 \mathrm{mmol})$ in dry THF (1 $\mathrm{mL})$ under a balloon pressure of $\mathrm{CO}$ was added substrate $\mathbf{5 a}(112 \mathrm{mg}, 0.5 \mathrm{mmol})$ in THF (10mL), and the reaction mixture was stirred at $50{ }^{\circ} \mathrm{C}$ for $24 \mathrm{~h}$. The solvent was removed under vacuum and the residue was purified by flash chromatograph on silica gel (first elution with petroleum ether/ethyl acetate $=6 / 1$, then with petrol ether/ethyl acetate $=2 / 1)$ to give compound $\mathbf{5 b}(23 \mathrm{mg})$ in $18 \%$ yield. ${ }^{1} \mathrm{H}$ NMR $\left(300 \mathrm{MHz}, \mathrm{CDCl}_{3}\right): \delta 1.72(\mathrm{t}, J=12.6 \mathrm{~Hz}, 1 \mathrm{H}), 1.78(\mathrm{~s}, 3 \mathrm{H}), 2.10(\mathrm{dd}, J=$ $3.3, J=18.0 \mathrm{~Hz}, 1 \mathrm{H}), 2.65(\mathrm{dd}, J=6.0,18.0 \mathrm{~Hz}, 1 \mathrm{H}), 2.80(\mathrm{dd}, J=7.5,12.6 \mathrm{~Hz}, 1 \mathrm{H}), 2.96(\mathrm{~m}, 1$ $\mathrm{H}), 3.21(\mathrm{~d}, J=18.9 \mathrm{~Hz}, 1 \mathrm{H}), 3.30(\mathrm{~d}, J=18.9 \mathrm{~Hz}, 1 \mathrm{H}), 3.78(\mathrm{~s}, 3 \mathrm{H}), 3.80(\mathrm{~s}, 3 \mathrm{H}) ;{ }^{13} \mathrm{C} \mathrm{NMR}$ $(75 \mathrm{MHz}, \mathrm{CDCl}): \delta 8.5,34.0,39.2,41.2,42.6,53.0,53.2,60.7,133.0,171.0,172.0,177.5,209.4$; LRMS $\left[\mathrm{C}_{13} \mathrm{H}_{16} \mathrm{O}_{5}\right]$, (EI) $\mathrm{m} / \mathrm{z}\left(\mathrm{M}^{+}\right)$, calcd 252, found 252.

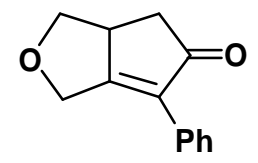

\section{Compound 6b}

To a solution of $\mathrm{PdCl}_{2}(13.3 \mathrm{mg}, 0.075 \mathrm{mmol})$, tmtu $(9.9 \mathrm{mg}, 0.075 \mathrm{mmol})$ in dry THF (1 $\mathrm{mL})$ under a balloon pressure of $\mathrm{CO}$ was added substrate $\mathbf{6 a}(86 \mathrm{mg}, 0.5 \mathrm{mmol})$ in THF $(10 \mathrm{~mL})$, and the reaction mixture was stirred at $50{ }^{\circ} \mathrm{C}$ for $24 \mathrm{~h}$. The solvent was removed under vacuum, and the residue was purified by flash chromatograph on silica gel (first elution with petroleum ether/ethyl acetate e $=6 / 1$, then with petrol ether/ethyl acetate $=2 / 1)$ to give $\mathbf{6 b}(40 \mathrm{mg})$ in $40 \%$ yield. ${ }^{1} \mathrm{H}$ NMR $\left(300 \mathrm{MHz}, \mathrm{CDCl}_{3}\right): \delta 2.37\left(\mathrm{dd}, J_{l}=3.6 \mathrm{~Hz}, J_{2}=18.0 \mathrm{~Hz}, 1 \mathrm{H}\right), 2.84\left(\mathrm{dd}, J_{l}=6.6\right.$ $\left.\mathrm{Hz}, J_{2}=18.0, \mathrm{~Hz} 1 \mathrm{H}\right), 3.23\left(\mathrm{dd}, J_{1}=7.2 \mathrm{~Hz}, J_{2}=11.4 \mathrm{~Hz}, 1 \mathrm{H}\right), 3.34(\mathrm{~m}, 1 \mathrm{H}), 4.38\left(\mathrm{dd}, J_{1}=7.2\right.$ $\left.\mathrm{Hz}, J_{2}=7.2 \mathrm{~Hz}, 1 \mathrm{H}\right), 4.61(\mathrm{~d}, J=16.2 \mathrm{~Hz}, 2 \mathrm{H}), 4.97(\mathrm{~d}, J=16.2 \mathrm{~Hz}, 1 \mathrm{H}), 7.34-7.56(\mathrm{~m}, 5 \mathrm{H})$; ${ }^{13} \mathrm{C}$ NMR $(75 \mathrm{MHz}, \mathrm{CDCl}): \delta 40.2,43.2,66.2,71.3,128.0,128.6,130.5,134.7,177.4,206.8$; LRMS $\left[\mathrm{C}_{13} \mathrm{H}_{12} \mathrm{O}_{2}\right],(\mathrm{EI}) \mathrm{m} / \mathrm{z}\left(\mathrm{M}^{+}\right)$: calcd 200, found 200 . 


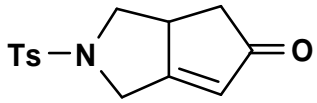

\section{Compound 9b}

To a solution of $\mathrm{PdCl}_{2}(13.3 \mathrm{mg}, 0.075 \mathrm{mmol})$, tmtu $(9.9 \mathrm{mg}, 0.075 \mathrm{mmol})$ in dry THF (1 $\mathrm{mL})$ under a balloon pressure of CO was added substrate $9 \mathbf{a}(124.5 \mathrm{mg}, 0.5 \mathrm{mmol})$ in THF (10mL), and the reaction mixture was stirred at $50{ }^{\circ} \mathrm{C}$ for $24 \mathrm{~h}$. The solvent was removed under vacuum, and the residue was purified by flash chromatograph on silica gel (petroleum ether/ethyl acetate $=$ $3 / 1)$ to give $9 \mathbf{b}(87 \mathrm{mg})$ in $63 \%$ yield. ${ }^{1} \mathrm{H} \mathrm{NMR}\left(300 \mathrm{MHz}, \mathrm{CDCl}_{3}\right): \delta 2.04\left(\mathrm{dd}, J_{1}=3.6, J_{2}=18 \mathrm{~Hz}\right.$, $5 \mathrm{H}), 2.37(\mathrm{~s}, 3 \mathrm{H}), 2.59\left(\mathrm{dd}, J_{l}=7.5, J_{2}=18 \mathrm{~Hz}, 1 \mathrm{H}\right), 2.66(\mathrm{t}, J=9.6 \mathrm{~Hz}, 1 \mathrm{H}), 3.16(\mathrm{~m}, 1 \mathrm{H})$, $4.00(\mathrm{t}, J=9.6 \mathrm{~Hz}, 1 \mathrm{H}), 4.06(\mathrm{~d}, J=16.8 \mathrm{~Hz}, 1 \mathrm{H}), 4.36(\mathrm{~d}, J=16.8 \mathrm{~Hz}, 1 \mathrm{H}), 5.99(\mathrm{~s}, 1 \mathrm{H}), 7.36$ $(\mathrm{d}, J=8.4 \mathrm{~Hz}, 2 \mathrm{H}), 7.74(\mathrm{~d}, J=8.4 \mathrm{~Hz}, 2 \mathrm{H}) ;{ }^{13} \mathrm{C} \mathrm{NMR}\left(75 \mathrm{MHz}, \mathrm{CDCl}_{3}\right): \delta 21.4,39.6,43.7,47.4$, 52.2, 126.0, 127.2, 129.8, 133.1, 144.0, 178.7, 207.4; LRMS $\left[\mathrm{C}_{14} \mathrm{H}_{15} \mathrm{NO}_{3} \mathrm{~S}\right]$, (EI) m/z $\left(\mathrm{M}^{+}\right)$, calcd 277 , found 277.

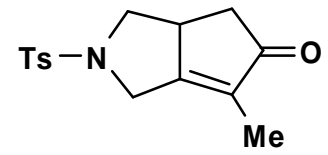

\section{Compound 10b}

To a solution of $\mathrm{PdCl}_{2}(13.3 \mathrm{mg}, 0.075 \mathrm{mmol})$, tmtu $(9.9 \mathrm{mg}, 0.075 \mathrm{mmol})$ in dry THF (1 $\mathrm{mL})$ under a balloon pressure of $\mathrm{CO}$ was added substrate 10a $(131.5 \mathrm{mg}, 0.5 \mathrm{mmol})$ in THF (10 $\mathrm{mL}$ ), and the reaction mixture was stirred at $50{ }^{\circ} \mathrm{C}$ for $36 \mathrm{~h}$. The solvent was removed under vacuum, and the residue was purified by flash chromatograph on silica gel (petroleum ether/ethyl acetate $=3 / 1)$ to give compound $\mathbf{1 0 b}(133.8 \mathrm{mg})$ in $92 \%$ yield. ${ }^{1} \mathrm{H} \mathrm{NMR}\left(300 \mathrm{MHz}, \mathrm{CDCl}_{3}\right): \delta 1.69$ (s, $3 \mathrm{H}), 2.07\left(\mathrm{dd}, J_{1}=3.3, J_{2}=18 \mathrm{~Hz}, 1 \mathrm{H}\right), 2.45(\mathrm{~s}, 3 \mathrm{H}), 2.44-2.65(\mathrm{~m}, 2 \mathrm{H}), 3.00(\mathrm{~m}, 1 \mathrm{H}), 4.00$ $(\mathrm{m}, 1 \mathrm{H}), 4.03(\mathrm{~d}, J=16.8 \mathrm{~Hz}, 1 \mathrm{H}), 4.22(\mathrm{~d}, J=16.8 \mathrm{~Hz}, 1 \mathrm{H}), 7.37(\mathrm{~d}, J=8.4 \mathrm{~Hz}, 2 \mathrm{H}), 7.76(\mathrm{~d}$, $J=8.4 \mathrm{~Hz}, 2 \mathrm{H}) ;{ }^{13} \mathrm{C} \mathrm{NMR}\left(75 \mathrm{MHz}, \mathrm{CDCl}_{3}\right): \delta 8.7,21.5,39.1,41.5,46.7,52.5,127.3,129.9$, 133.5, 134.0, 171.0, 207.6; LRMS $\left[\mathrm{C}_{15} \mathrm{H}_{17} \mathrm{NO}_{3} \mathrm{~S}\right]$, (EI) $\mathrm{m} / \mathrm{z}\left(\mathrm{M}^{+}\right)$, calcd 291, found 291.

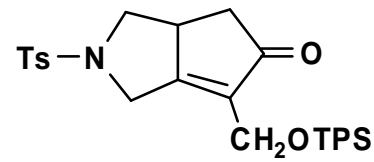

\section{Compound 11b}

To a solution of $\mathrm{PdCl}_{2}(13.3 \mathrm{mg}, 0.075 \mathrm{mmol})$, tmtu $(9.9 \mathrm{mg}, 0.075 \mathrm{mmol})$ in dry THF (1 $\mathrm{ml}$ ) under a balloon pressure of CO was added substrate 11a $(259 \mathrm{mg}, 0.5 \mathrm{mmol})$ in THF $(10 \mathrm{~mL})$, and the reaction mixture was stirred at $50{ }^{\circ} \mathrm{C}$ for $36 \mathrm{~h}$. The solvent was removed under vacuum and the residue was purified by flash chromatograph on silica gel (petrol ether/ethyl acetate $=3 / 1$ ) to give 11b $(243 \mathrm{mg})$ in $89 \%$ yield. ${ }^{1} \mathrm{H}$ NMR $\left(300 \mathrm{MHz}, \mathrm{CDCl}_{3}\right): 1.08(\mathrm{~s}, 9 \mathrm{H}), 2.02\left(\mathrm{dd}, J_{l}=3.6 \mathrm{~Hz}\right.$, $\left.J_{2}=18.0 \mathrm{~Hz}, 1 \mathrm{H}\right), 2.46(\mathrm{~s}, 3 \mathrm{H}), 2.42-2.58(\mathrm{~m}, 3 \mathrm{H}), 3.10(\mathrm{~m}, 1 \mathrm{H}), 4.02(\mathrm{t}, J=8.4 \mathrm{~Hz}, 1 \mathrm{H}), 4.30-$ $4.44(\mathrm{~m}, 4 \mathrm{H}), 7.25-7.7 .47(\mathrm{~m}, 8 \mathrm{H}), 7.52-7.63(\mathrm{~m}, 4 \mathrm{H}), 7.65(\mathrm{~d}, J=6.6 \mathrm{~Hz}, 2 \mathrm{H}) ;{ }^{13} \mathrm{C} \mathrm{NMR}$ $\left(75 \mathrm{MHz}, \mathrm{CDCl}_{3}\right): \delta 19.0,21.5,26.8,39.5,42.5,47.9,52.2,59.4,127.5,127.8,127.9,129.8,129.9$, $130.0,132.2,132.4,132.9,135.3,135.4,136.7,143.8,171.9,205.7$; LRMS $\left[\mathrm{C}_{31} \mathrm{H}_{35} \mathrm{NO}_{4} \mathrm{SSi}\right]$, (EI) $\mathrm{m} / \mathrm{z}\left(\mathrm{M}^{+}+1\right)$, calcd 546 , found 546 . 


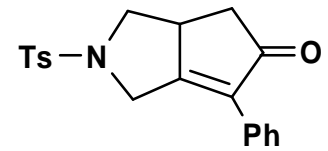

\section{Compound 12b}

To a solution of $\mathrm{PdCl}_{2}(13.3 \mathrm{mg}, 0.075 \mathrm{mmol})$, tmtu $(9.9 \mathrm{mg}, 0.075 \mathrm{mmol})$ in dry THF (1 $\mathrm{mL})$ under a balloon pressure of $\mathrm{CO}$ was added substrate 12a $(162.8 \mathrm{mg}, 0.5 \mathrm{mmol})$ in THF (10 $\mathrm{mL}$ ), and the reaction mixture was stirred at $50{ }^{\circ} \mathrm{C}$ for $48 \mathrm{~h}$. The solvent was removed under vacuum and the residue was purified by flash chromatograph on silica gel (first elution with petroleum ether/ethyl acetate $=6 / 1$, then with petrol ether/ethyl acetate $=2 / 1)$ to give $\mathbf{1 2 b}(102.5$ $\mathrm{mg}$ ) in $58 \%$ yield. ${ }^{1} \mathrm{H}$ NMR $(300 \mathrm{MHz}):, \delta 1.57(\mathrm{~s}, 3 \mathrm{H}), 2.28\left(\mathrm{dd}, J_{l}=3.9 \mathrm{~Hz}, J_{2}=18.0 \mathrm{~Hz}, 1 \mathrm{H}\right)$, $2.41(\mathrm{~s}, 3 \mathrm{H}), 2.62\left(\mathrm{dd}, J_{1}=9.3 \mathrm{~Hz}, J_{2}=11.1 \mathrm{~Hz}, 1 \mathrm{H}\right), 2.82\left(\mathrm{dd}, J_{l}=6.6 \mathrm{~Hz}, J_{2}=18.0 \mathrm{~Hz}, 1 \mathrm{H}\right)$, $3.2(\mathrm{~m}, 1 \mathrm{H}), 4.05-4.08(\mathrm{~m}, 2 \mathrm{H}), 4.66\left(\mathrm{dd}, J_{l}=1.8 \mathrm{~Hz}, J_{2}=17.1 \mathrm{~Hz}, 1 \mathrm{H}\right), 7.38(\mathrm{~d}, J=6.6 \mathrm{~Hz}, 2$ $\mathrm{H}), 7.40-7.48(\mathrm{~m}, 5 \mathrm{H}), 7.74(\mathrm{~d}, J=6.6 \mathrm{~Hz}, 2 \mathrm{H}) ;{ }^{13} \mathrm{C} \mathrm{NMR}\left(75 \mathrm{MHz}, \mathrm{CDCl}_{3}\right): \delta 21.2,40.5,41.6$, 48.1, 51.7, 127.2, 128.0, 128.5, 128.8, 129.8, 129.9, 133.6, 135.8, 143.9, 172.0, 205.5; LRMS $\left[\mathrm{C}_{20} \mathrm{H}_{19} \mathrm{NO}_{3} \mathrm{~S}\right]$, (EI) $\mathrm{m} / \mathrm{z}\left(\mathrm{M}^{+}\right)$, calcd 353, found 353 .

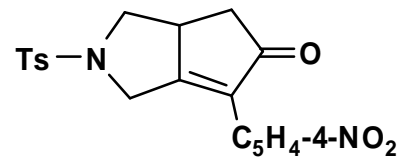

\section{Compound 13b}

To a solution of $\mathrm{PdCl}_{2}(13.3 \mathrm{mg}, 0.075 \mathrm{mmol})$, tmtu $(9.9 \mathrm{mg}, 0.075 \mathrm{mmol})$ in dry THF (1 $\mathrm{mL}$ ) under a balloon pressure of CO was added substrate $13 \mathbf{a}(185 \mathrm{mg}, 0.5 \mathrm{mmol})$ in THF $(10 \mathrm{~mL})$, and the reaction mixture was stirred at $50{ }^{\circ} \mathrm{C}$ for $48 \mathrm{~h}$. The solvent was removed under vacuum and the residue was purified by flash chromatograph on silica gel (petrol ether/ethyl acetate $=2 / 1$ ) to give 13b (179 mg) in 90\% yield. ${ }^{1} \mathrm{H}$ NMR (300MHz, DMSO): $\delta 2.33(\mathrm{~s}, 3 \mathrm{H}), 2.45$ (dd, $J_{l}=3.6$ $\left.\mathrm{Hz}, J_{2}=18.0 \mathrm{~Hz}, 1 \mathrm{H}\right), 2.70(\mathrm{~m}, 2 \mathrm{H}), 3.19(\mathrm{~m}, 2 \mathrm{H}), 3.93(\mathrm{t}, J=9.0 \mathrm{~Hz}, 1 \mathrm{H}), 4.03(\mathrm{~d}, J=18.0 \mathrm{~Hz}$, $2 \mathrm{H}), 4.91(\mathrm{~d}, 18.0 \mathrm{~Hz}), 7.37$ (d, $J=8.4 \mathrm{~Hz}, 2 \mathrm{H}), 7.73(\mathrm{~d}, J=8.4 \mathrm{~Hz}, 2 \mathrm{H}), 7.74(\mathrm{~d}, J=8.4 \mathrm{~Hz}, 2$ $\mathrm{H}), 8.23(\mathrm{~d}, J=8.4 \mathrm{~Hz}, 2 \mathrm{H}) ;{ }^{13} \mathrm{C}$ NMR $(75 \mathrm{MHz}, \mathrm{DMSO}): \delta 20.9,40.4,42.1,48.5,51.2,123.6$, $127.4,129.2,130.1,132.5,133.2,136.6,143.8,146.9,177.6,205.2$; LRMS $\left[\mathrm{C}_{19} \mathrm{H}_{18} \mathrm{~N}_{2} \mathrm{O}_{5} \mathrm{~S}\right]$, (EI) $\mathrm{m} / \mathrm{z}\left(\mathrm{M}^{+}\right)$, calcd 398, found 398 .

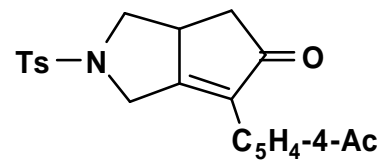

\section{Compound 14b}

To a solution of $\mathrm{PdCl}_{2}(13.3 \mathrm{mg}, 0.075 \mathrm{mmol}), \mathrm{tmtu}(9.9 \mathrm{mg}, 0.075 \mathrm{mmol})$ in dry THF (1 $\mathrm{mL}$ ) under a balloon pressure of $\mathrm{CO}$ was added substrate $\mathbf{1 4 a}(183.5 \mathrm{mg}, 0.5 \mathrm{mmol})$ in THF (10 $\mathrm{mL}$ ), and the reaction mixture was stirred at $50{ }^{\circ} \mathrm{C}$ for $48 \mathrm{~h}$. The solvent was removed under vacuum and the residue was purified by flash chromatography on silica gel (petrol ether/ethyl acetate $=3 / 1)$ to give compound $\mathbf{1 4 b}(126.4 \mathrm{mg})$ in $64 \%$ yield. ${ }^{1} \mathrm{H}$ NMR $\left(300 \mathrm{MHz}, \mathrm{CDCl}_{3}\right): \delta 2.25$ $\left(\mathrm{dd}, J_{I}=3.9 \mathrm{~Hz}, J_{2}=18.0 \mathrm{~Hz}, 1 \mathrm{H}\right), 2.41(\mathrm{~s}, 3 \mathrm{H}), 2.67(\mathrm{~s}+\mathrm{m}, 4 \mathrm{H}), 2.85\left(\mathrm{dd}, J_{I}=6.3 \mathrm{~Hz}, J_{2}=18\right.$ $\mathrm{Hz}, 1 \mathrm{H}), 3.26(\mathrm{~m}, 2 \mathrm{H}), 4.07(\mathrm{~d}, J=17.1 \mathrm{~Hz}, 1 \mathrm{H}), 4.10(\mathrm{t}, J=8.4 \mathrm{~Hz}, 1 \mathrm{H}), 4.70(\mathrm{~d}, J=17.1 \mathrm{~Hz})$, 
$7.28(\mathrm{~d}, J=8.4 \mathrm{~Hz}, 2 \mathrm{H}), 7.58(\mathrm{~d}, J=6.9 \mathrm{~Hz}, 2 \mathrm{H}), 7.74(\mathrm{~d}, J=6.9 \mathrm{~Hz}, 2 \mathrm{H}), 8.02(\mathrm{~d}, J=8.4 \mathrm{~Hz}, 2$ $\mathrm{H}) ;{ }^{13} \mathrm{C}$ NMR $\left(75 \mathrm{MHz}, \mathrm{CDCl}_{3}\right): \delta 21.6,26.7,40.7,42.2,48.4,51.9,127.4,128.3,128.5,130.1$, 133.4, 134.3, 135.2, 136.9, 144.2, 174.0, 197.5, 204.9; LRMS $\left[\mathrm{C}_{22} \mathrm{H}_{21} \mathrm{NO}_{4} \mathrm{~S}\right]$, (EI) m/z $\left(\mathrm{M}^{+}\right)$, calcd 395 , found 395 .

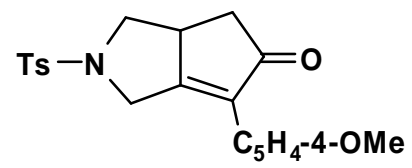

\section{Compound 15b}

To a solution of $\mathrm{PdCl}_{2}(13.3 \mathrm{mg}, 0.075 \mathrm{mmol})$, tmtu (9.9 mg, $\left.0.075 \mathrm{mmol}\right)$ in dry THF (1 $\mathrm{mL}$ ) under a balloon pressure of CO was added substrate $15 \mathrm{a}(177.5 \mathrm{mg}, 0.5 \mathrm{mmol})$ inTHF (10 $\mathrm{mL}$ ), and the reaction mixture was stirred at $50{ }^{\circ} \mathrm{C}$ for $48 \mathrm{~h}$. The solvent was removed under vacuum and the residue was purified by flash chromatograph on silica gel (petrol ether/ethyl acetate $=3 / 1)$ to give $\mathbf{1 5 b}(82 \mathrm{mg})$ in $43 \%$ yield. ${ }^{1} \mathrm{H}$ NMR $\left(300 \mathrm{MHz}, \mathrm{CDCl}_{3}\right): \delta 2.20\left(\mathrm{dd}, J_{I}=3.6\right.$ $\left.\mathrm{Hz}, J_{2}=17.7 \mathrm{~Hz}, 1\right), 2.43(\mathrm{~s}, 3), 2.58(\mathrm{t}, J=10.2 \mathrm{~Hz} 1), 2.78\left(\mathrm{dd}, J_{l}=3.0 \mathrm{~Hz}, J_{2}=18 \mathrm{~Hz}, 1 \mathrm{H}\right)$, $4.05(\mathrm{~m}, 2 \mathrm{H}), 4.64(\mathrm{~d}, J=16.8 \mathrm{~Hz}, 1 \mathrm{H}), 6.92(\mathrm{~d}, J=9.0 \mathrm{~Hz}, 2 \mathrm{H}), 7.30(\mathrm{~d}, J=8.1 \mathrm{~Hz}, 2 \mathrm{H}), 7.45$ $(\mathrm{d}, J=9.0 \mathrm{~Hz}, 2 \mathrm{H}), 7.73(\mathrm{~d}, J=8.1 \mathrm{~Hz}, 2 \mathrm{H}) ;{ }^{13} \mathrm{C} \mathrm{NMR}\left(75 \mathrm{MHz}, \mathrm{CDCl}_{3}\right): \delta 21.5,40.6,41.6,48.3$, 51.9, 55.2, 114.0, 122.4, 127.3, 129.5, 129.9, 133.5, 135.4, 143.9, 159.9, 169.8, 205.9; LRMS $\left[\mathrm{C}_{21} \mathrm{H}_{2} \mathrm{NO}_{4} \mathrm{~S}\right]$, (EI) $\mathrm{m} / \mathrm{z}\left(\mathrm{M}^{+}\right)$, calcd 383, found 383 .

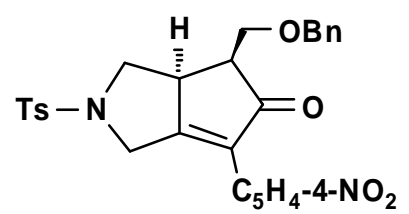

\section{Compound 16b}

To a solution of $\mathrm{PdCl}_{2}(13.3 \mathrm{mg}, 0.075 \mathrm{mmol})$, tmtu $(9.9 \mathrm{mg}, 0.075 \mathrm{mmol})$ in dry THF (1 $\mathrm{mL}$ ) under a balloon pressure of CO was added substrate 16a $(245 \mathrm{mg}, 0.5 \mathrm{mmol})$ in THF (10 mL), and the reaction mixture was stirred at $50{ }^{\circ} \mathrm{C}$ for $48 \mathrm{~h}$. The solvent was removed under vacuum and the residue was purified by flash chromatograph on silica gel (petroleum ether/ethyl acetate $=3 / 1$ ) to give $\mathbf{1 6 b}(207 \mathrm{mg})$ in $80 \%$ yield. ${ }^{1} \mathrm{H}$ NMR $\left(300 \mathrm{MHz}, \mathrm{CDCl}_{3}\right): \delta 2.36(\mathrm{~s}, 1 \mathrm{H}), 2.43(\mathrm{~s}, 3 \mathrm{H}), 3.07$ $(\mathrm{m}, 1 \mathrm{H}), 3.55(\mathrm{~m}, 1 \mathrm{H}), 3.61\left(\mathrm{dd}, J_{l}=8.1 \mathrm{~Hz}, J_{2}=9.6 \mathrm{~Hz}, 1 \mathrm{H}\right), 3.71\left(\mathrm{dd}, J_{l}=3.6 \mathrm{~Hz}, J_{2}=9.6 \mathrm{~Hz}\right.$, $1 \mathrm{H}), 3.99(\mathrm{t}, J=9.0 \mathrm{~Hz}, 1 \mathrm{H}), 4.04(\mathrm{~d}, J=17.4 \mathrm{~Hz}, 1 \mathrm{H}), 4.40(\mathrm{~m}, 2 \mathrm{H}), 4.67(\mathrm{~d}, J=17.4 \mathrm{~Hz}, 1 \mathrm{H})$, $7.12(\mathrm{~m}, 2 \mathrm{H}), 7.24(\mathrm{~m}, 5 \mathrm{H}), 7.63(\mathrm{~d}, J=8.7 \mathrm{~Hz}, 2 \mathrm{H}), 7.69(\mathrm{~d}, J=8.1 \mathrm{~Hz}, 2 \mathrm{H}), 8.24(\mathrm{~d}, J=8.1$ $\mathrm{Hz}, 2 \mathrm{H}) ;{ }^{13} \mathrm{C}$ NMR $\left(75 \mathrm{MHz}, \mathrm{CDCl}_{3}\right): \delta 21.5,45.6,47.8,48.4,49.6,67.9,73.2,123.7,127.3$, $127.4,127.8,128.4,128.8,129.9,133.3,133.4,136.2,137.1,144.1,147.4,176.3,204.9$; LRMS $\left[\mathrm{C}_{28} \mathrm{H}_{26} \mathrm{~N}_{2} \mathrm{O}_{6} \mathrm{~S}\right],(\mathrm{EI}) \mathrm{m} / \mathrm{z}\left(\mathrm{M}^{+}\right)$, calcd 518, found 518 .

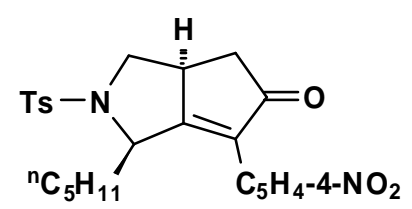

Compound 17b 
To a solution of $\mathrm{PdCl}_{2}(13.3 \mathrm{mg}, 0.075 \mathrm{mmol})$, tmtu $(9.9 \mathrm{mg}, 0.075 \mathrm{mmol})$ in dry THF (1 $\mathrm{mL})$ under a balloon pressure of $\mathrm{CO}$ was added substrate 17a $(220 \mathrm{mg}, 0.5 \mathrm{mmol})$ in $1 \mathrm{THF}(10$ $\mathrm{mL}$ ), and the reaction mixture was stirred at $50{ }^{\circ} \mathrm{C}$ for $36 \mathrm{~h}$. The solvent was removed under vacuum and the residue was purified by flash chromatograph on silica gel (petrol ether/ethyl acetate $=3 / 1)$ to give $\mathbf{1 7 b}(225 \mathrm{mg})$ in $96 \%$ yield. ${ }^{1} \mathrm{H}$ NMR $\left(200 \mathrm{MHz}, \mathrm{CDCl}_{3}\right): \delta 0.68(\mathrm{~m}, 3 \mathrm{H})$, 0.91-0.99 (m, 8 H), 1.19-1.44 (m, $2 \mathrm{H}), 1.77$ (m, $1 \mathrm{H}), 2.23$ (m, $1 \mathrm{H}), 2.46$ (s, $3 \mathrm{H}), 2.68$ (m, $2 \mathrm{H})$, $2.92(\mathrm{t}, J=11.6 \mathrm{~Hz}, 1 \mathrm{H}), 4.15\left(\mathrm{dd}, J_{l}=7.6 \mathrm{~Hz}, J_{2}=11.6 \mathrm{~Hz}, 1 \mathrm{H}\right), 5.11(\mathrm{t}, J=4.0 \mathrm{~Hz}, 1 \mathrm{H}), 7.36$ $(\mathrm{d}, J=8.0 \mathrm{~Hz}, 2 \mathrm{H}), 7.49$ (d, $J=9.2 \mathrm{~Hz}, 2 \mathrm{H}), 7.83(\mathrm{~d}, J=8.0 \mathrm{~Hz}, 2 \mathrm{H}), 8.29$ (d, $J=9.2 \mathrm{~Hz}, 2 \mathrm{H})$; ${ }^{13} \mathrm{C}$ NMR $\left(50 \mathrm{MHz}, \mathrm{CDCl}_{3}\right): \delta 13.7,21.4,21.9,23.6,31.0,31.4,40.2,43.6,51.9,60.1,123.4$, 126.9, 129.9, 130.1, 135.3, 135.7, 136.4, 144.1, 147.4, 180.7, 204.7; LRMS [C $\left.{ }_{25} \mathrm{H}_{28} \mathrm{~N}_{2} \mathrm{O}_{5} \mathrm{~S}\right]$, (EI) $\mathrm{m} / \mathrm{z}\left(\mathrm{M}^{+}\right)$, calcd 468, found 468 .

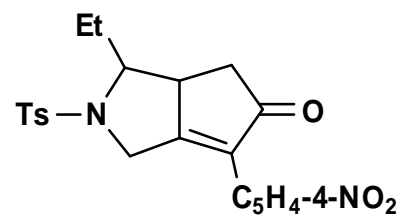

\section{Compound 18b}

To a solution of $\mathrm{PdCl}_{2}(13.3 \mathrm{mg}, 0.075 \mathrm{mmol})$, tmtu $(9.9 \mathrm{mg}, 0.075 \mathrm{mmol})$ in dry THF (1 $\mathrm{mL})$ under a balloon pressure of CO was added substrate 18a (199 mg, $0.5 \mathrm{mmol})$ in THF $10 \mathrm{~mL})$, and the reaction mixture was stirred at $50{ }^{\circ} \mathrm{C}$ for $48 \mathrm{~h}$. The solvent was removed under vacuum and the residue was purified by flash chromatograph on silica gel (petrol ether/ethyl acetate $=3 / 1$ ) to give $18 \mathrm{~b}(196 \mathrm{mg})$ in $92 \%$ yield. ${ }^{1} \mathrm{H}$ NMR $\left(200 \mathrm{MHz}, \mathrm{CDCl}_{3}\right): \delta 0.96(\mathrm{t}, J=7.2 \mathrm{~Hz}, 3 \mathrm{H}), 1.86(\mathrm{~m}$, $1 \mathrm{H}), 2.19-2.37(\mathrm{~m}, 2 \mathrm{H}), 2.46(\mathrm{~s}, 3 \mathrm{H}), 2.82\left(\mathrm{dd}, J_{l}=6.4 \mathrm{~Hz}, J_{2}=18 \mathrm{~Hz}, 1 \mathrm{H}\right), 2.92-3.13(\mathrm{~m}, 2 \mathrm{H})$, $4.32(\mathrm{~d}, J=17.2 \mathrm{~Hz}, 1 \mathrm{H}), 4.58(\mathrm{~d}, J=17.2 \mathrm{~Hz}, 1 \mathrm{H}), 7.14(\mathrm{~d}, J=8.6 \mathrm{~Hz}, 2 \mathrm{H}), 7.53(\mathrm{~d}, J=8.8 \mathrm{~Hz}$, $2 \mathrm{H}), 7.57(\mathrm{~d}, J=8.6 \mathrm{~Hz}, 2 \mathrm{H}), 8.30(\mathrm{~d}, J=8.8 \mathrm{~Hz}, 2 \mathrm{H}) ; \mathrm{LRMS}\left[\mathrm{C}_{22} \mathrm{H}_{22} \mathrm{~N}_{2} \mathrm{O}_{5} \mathrm{~S}\right],(\mathrm{EI}) \mathrm{m} / \mathrm{z}\left(\mathrm{M}^{+}\right)$, calcd 426, found 426.

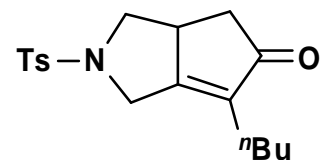

\section{Compound 19b}

To a solution of $\mathrm{PdCl}_{2}(13.3 \mathrm{mg}, 0.075 \mathrm{mmol})$, tmtu $(9.9 \mathrm{mg}, 0.075 \mathrm{mmol})$ in dry THF (1 $\mathrm{mL})$ under a balloon pressure of $\mathrm{CO}$ was added substrate $19 \mathbf{a}(153 \mathrm{mg}, 0.5 \mathrm{mmol})$ in THF $(10 \mathrm{~mL})$, and the reaction mixture was stirred at $50{ }^{\circ} \mathrm{C}$ for $48 \mathrm{~h}$. The solvent was removed under vacuum and the residue was purified by flash chromatograph on silica gel (petrol ether/ethyl acetate $=3 / 1$ ) to give $19 \mathrm{~b}(97 \mathrm{mg})$ in $58 \%$ yield. ${ }^{1} \mathrm{H}$ NMR $\left(300 \mathrm{MHz}, \mathrm{CDCl}_{3}\right): \delta 0.86(\mathrm{t}, 7.2 \mathrm{~Hz}), 1.21-1.30(\mathrm{~m}, 4 \mathrm{H})$, $2.06(\mathrm{~m}, 3 \mathrm{H}), 2.16(\mathrm{~m}, 1 \mathrm{H}), 2.44(\mathrm{~s}, 3 \mathrm{H}), 2.64(\mathrm{~m}, 2 \mathrm{H}), 3.0(\mathrm{~m}, 1 \mathrm{H}), 3.99(\mathrm{~m}, 2 \mathrm{H}), 4.28(\mathrm{~d}, J=$ $15.9 \mathrm{~Hz}, 1 \mathrm{H}), 7.37(\mathrm{~d}, J=8.4 \mathrm{~Hz}, 2 \mathrm{H}), 7.76(\mathrm{~d}, J=8.4 \mathrm{~Hz}, 2 \mathrm{H}) ;{ }^{13} \mathrm{C} \mathrm{NMR}\left(75 \mathrm{MHz}, \mathrm{CDCl}_{3}\right): \delta$ 13.7, 21.9, 22.5, 23.6, 29.7, 39.3, 41.7, 46.9, 52.6, 127.4, 129.9, 133,4, 138.4, 144.0, 170.7, 207.5; LRMS $\left[\mathrm{C}_{18} \mathrm{H}_{23} \mathrm{NO}_{3} \mathrm{~S}\right]$, (EI) m/z $\left(\mathrm{M}^{+}\right)$, calcd 333, found 333 . 